\title{
Concordance Between Persons with Multiple Sclerosis and Treating Physician on Medication Effects and Health Status
}

\author{
Efrat Neter (iD) \\ Lea Glass-Marmor ${ }^{2}$ \\ Loren Haiien ${ }^{2}$ \\ Ariel Miller ${ }^{2,3}$ \\ 'Department of Behavioral Sciences, \\ Ruppin Academic Center, Emeq Hefer, \\ Israel; ${ }^{2}$ Multiple Sclerosis Center \& \\ Department of Neurology, Carmel \\ Medical Center, Haifa, Israel; ${ }^{3}$ Rappaport \\ Faculty of Medicine, Technion Institute of \\ Technology, Haifa, Israel
}

\begin{abstract}
Background: As the number of treatment options for multiple sclerosis (MS) has expanded, alignment between physician and patient on effects of medication has emerged as important for medication persistence/discontinuation.

Objective: To evaluate physician-patient agreement levels on medication effect and health status.

Methods: Persons with MS (PwMS) ( $\mathrm{n}=71)$ participated in a cross-sectional study collecting their satisfaction (using the Treatment Satisfaction Questionnaire for Medication), intention to dis/continue treatment and global health perception; physicians assessed response to medication and global health status.

Results: Concordance between PwMS' assessment of medication effectiveness and physician's assessment on response to medication, health status and EDSS were $r_{\mathrm{s}}=0.50, r_{\mathrm{s}}=0.57$ and $r_{\mathrm{s}}=-0.58$, respectively.

Conclusion: The significant concordance attests to physician-patient effective communication and may contribute to improved medication adherence.
\end{abstract}

Keywords: concordance, disease-modifying therapy, patient-centered approach, multiple sclerosis, patient-reported outcomes, participatory medicine

\section{Introduction}

Shared medical decision-making and patient engagement have gained recognition and acceptance in the treatment of multiple sclerosis (MS), ${ }^{1-3}$ where no single treatment path is recommended. ${ }^{4}$ Their relevance has increased as the number of treatment options for relapsing MS has expanded in the past decade, ${ }^{5}$ concomitant with adverse side effects, partial adherence to therapy regime and discontinuation. ${ }^{1,6}$ Thus, concordance on treatment effectiveness between the physician and the patient has emerged as important for treatment alliance. $^{4,7}$

Few studies evaluated directly the agreement between persons with chronic illnesses and physicians' assessments, most of these studies targeting health-related quality of life $^{8}$ or treatment preferences. ${ }^{4}$ In MS, divergent perceptions between physicians and persons with multiple sclerosis (PwMS) were mostly noted. ${ }^{8-10}$ For example, PwMS reported a significantly worse perception of MS as a malignant disease than their treating physician and they were more willing to continue treatment with a specific medication. ${ }^{10}$ Alongside, PwMS disagreed with the physician which health
Correspondence: Efrat Neter

Department of Behavioral Sciences,

Ruppin Academic Center, 3 Bait, Emeq

Hefer, 4025000, Israel

Tel +972-54-6462677

Fax +972-9-8983043

Email neter@ruppin.ac.il 
domains are more important ${ }^{9}$ and the only significant association was recorded in bodily pain and social functioning. ${ }^{8}$

As higher congruence between PwMS' and physicians' perceptions was associated with persistence on medication, ${ }^{11}$ concordance in perceptions becomes even more crucial and may turn into a resource in confronting partial adherence and discontinuation of treatment. The present study's goal was to investigate concordance in the perceptions on health status and medication, focusing on effectiveness, between PwMS and their treating neurologist.

\section{Methods}

\section{Participants and Design}

PwMS ( $\mathrm{n}=71)$ attending an outpatient MS clinic at Carmel Medical Center, Haifa, treated with DMTs in the modalities of infusion $(n=23)$, injection $(n=12)$ and oral $(n=36)$ for 1-3 years' duration and their treating neurologist were included. The design was cross-sectional. The participating physicians $(\mathrm{n}=2)$ were MS specialized neurologists at the MS clinic and the recruitment of PwMS was according to the following criteria: age $>18$ and $1-3$ years duration on current medication. This study was approved by the Ethical Committee at Carmel Medical Center (\# 0034-13CMC). Participants were informed about the study protocol and signed informed consent. Confidentiality and anonymity were assured to all enrolled participants. PwMS filled the survey blind to its filling by another party.

\section{Measures}

The Treatment Satisfaction Questionnaire for Medication version 1.4 (TSQM 1.4) ${ }^{12}$ is a patient-reported outcome (PRO) measure containing 14 items tapping four domains: effectiveness; side effects; convenience; and global satisfaction. Each domain's score is on a 0-100 scale, with higher values indicating higher satisfaction, better perceived effectiveness, better convenience and lower perceived side effects.

Self-rated health (SRH) was measured using a single question; respondents evaluated their health on a five-point response scale ranging from "excellent" to "poor". PwMS were also asked about their wish to dis/continue with their current medication, responding on a five-point scale.

Physician's assessments were tapped by two items recorded on a special form, specific to the study. The first item assessed the PwMS' global health status on a three-point scale (improved-stable-worse). The second item assessed PwMS' response to medication, based in clinical indicators; the response was on a three-point scale (good-medium-not good). This measurement, thought subjective and different from the survey the PwMS filled, was chosen as characteristic of routine medical documentation in PwMS' files. The Expanded Disability Status Scale (EDSS) $)^{13}$ was also recorded.

\section{Statistical Analyses}

Descriptive statistics were performed, characterizing the PwMS and the main variables. Then, as physician's assessments were on an ordinal scale, Spearman $\rho$ correlations between perceptions of PwMS and the physician were computed. A two-sided $p$ value $<0.05$ was taken to indicate statistical significance. Correlation size $<0.1$ was interpreted as negligible, $0.1-0.39$ as low, $0.40-0.69$ as moderate and 0.70 onward as strong.

\section{Results}

PwMS ( $\mathrm{n}=71)$ were mostly women $(\mathrm{n}=42,59.2 \%)$, the mean age was $41.9(\mathrm{SD}=12.9)$, most were married $(\mathrm{n}=47$, $66.2 \%)$ and either working or studying $(\mathrm{n}=59,83.0 \%)$. Their physical disability score was moderate $(M=3.5$, $S D=2.5, I Q R=1.0-6.0)$ and the mean MS duration was $M=8.2(S D=14.2)$.

The assessments of PwMS and the physician are presented in Table 1. The highest score in PwMS' rating of satisfaction was assigned to convenience $(M=73.7$, $S D=22.7)$, then to (lack of) side effects $(M=68.3$, $S D=26.3)$, and least to effectiveness $(M=58.3, S D=23.8)$ and global evaluation $(M=60.1, S D=25.1)$. Still, most PwMS reported wanting to continue their medication $(M=4.0, S D=1.1)$ and rated their health as moderate $(M=2.7, S D=1.0)$. The physician's evaluation was that more than a third's health status worsened $(n=26,36.6 \%)$ while about a half were stable $(\mathrm{n}=39,54.9 \%)$; the response to the medication was good among half of the PwMS $(50.7 \%)$ and medium or not good among $18.3 \%$ and $23.9 \%$, respectively.

Table 2 presents the Spearman correlations between the assessments of PwMS and the physician on the health status and the medication. The PwMs' assessments regarding medication are all positively significantly associated, with high association between global, effectiveness and wanting to continue the medication (range: $0.60-0.75$ ). The physician's assessments are also significantly associated: the response to medication with the general health condition $\left(r_{\mathrm{s}}=0.81\right.$, 
Table I Assessments by PwMS ( $\mathrm{n}=7 \mathrm{I})$ and Physicians, (Mean $(\mathrm{SD}) / \mathrm{n}(\%))$

\begin{tabular}{|l|l|l|}
\hline Variable & Mean (SD) & n (\%) \\
\hline PwMS' assessments & & \\
Medication effectiveness & $58.3(23.8)$ & \\
Medication side effects & $68.3(26.3)$ & \\
Medication convenience & $73.7(22.7)$ & \\
Medication global & $60.1(25.1)$ & \\
Want to continue medication & $4.0(1.1)$ & \\
Self-rated health, I-5 scale & $2.7(1.0)$ & \\
\hline Physician's assessments & & \\
Response to medication & & $36(50.7)$ \\
Good & & $13(18.3)$ \\
Medium & & $17(23.9)$ \\
Not good & & $5(7.0)$ \\
\hline Missing & & $2(2.8)$ \\
\hline Health status & & $39(54.9)$ \\
Improved & & $26(36.6)$ \\
Stable & & $4(5.6)$ \\
Worse & & \\
Missing & & \\
\hline Expanded Disability Status Scale & $3.5(2.5)$ & \\
\hline
\end{tabular}

$p<0.001)$ and these assessments are associated moderately negatively with EDSS, $r_{\mathrm{s}}=-0.64$ and $r_{\mathrm{s}}=-0.54$, respectively.

The concordance between assessments of the physician and PwMS regarding global health status are significant $\left(r_{\mathrm{s}}\right.$ $=0.53, p<0.001)$ as well as the concordance on the medication: $r_{\mathrm{s}}=0.50$ and $r_{\mathrm{s}}=0.45$, for effectiveness and global, respectively. These two focal associations between PwMS perceptions on effectiveness and the physician's evaluation on response to medication were significant also when corrected for multiple testing ( $p$ values $<0.05$ ). PwMS assessments of side effects were not significantly associated with the physician's assessment of the response to medication $\left(r_{\mathrm{s}}\right.$
$=0.09, p>0.05)$ and convenience was weakly associated with the physician's assessment $\left(r_{\mathrm{s}}=0.26, p<0.05\right)$.

\section{Discussion}

This is one of the first studies in MS documenting concordance on medication-related perceptions between PwMS and their neurologist. The significant moderate concordance was evident when both parties evaluated the same domains (ie, health status, medication effectiveness, range 0.50-0.53) indicating convergent validity. The level of agreement was much lower and weaker when the neurologist and the PwMS evaluated related, yet dissimilar domains, such as the neurologist evaluating effectiveness and the PwMS evaluating side effects or wanting to (dis)continue the medication (range $0.08-0.38$ ), thereby showing divergent validity. This concordance on medication-related perceptions is in contrast to the divergence found on most aspects in quality of life. ${ }^{8}$

This significant concordance may be interpreted as reflecting continued two-sided effective communication between PwMS and their neurologist along the treatment course: discussing treatment options (initiate a medication, discontinue, switch medication), potential consequences of options, test results, reported symptoms and side effects experienced by the PwMS. It may also reflect the undergoing shift in paradigm from treating the disease to treating the patient and is especially important when considering the increasing number of effective treatment options for MS, on the one hand, and the information on the Internet and social media available to people with chronic illnesses, on the other. ${ }^{14}$ Personalized information, effective communication, shared decisionmaking and high concordance between PwMS and physicians may also contribute to medication adherence and persistence.

Although medication adherence was not assessed in this study, interest in dis/continue the medication, a variable alighted with adherence, was found associated

Table 2 Spearman Correlations Between Assessments of PwMS $(n=66-7 I)$ and Physicians

\begin{tabular}{|c|c|c|c|c|c|c|c|c|c|}
\hline & $\mathbf{I}$ & 2 & 3 & 4 & 5 & 6 & 7 & 8 & 9 \\
\hline I. PwMS: Medic. effectiveness & & - & & & & & & & \\
\hline 2. PwMS: Medic. side effects & 0.02 & - & & & & & & & \\
\hline 3. PwMS: Medic. convenience & $0.34 * *$ & $0.37 * *$ & - & & & & & & \\
\hline 4. PwMS: Medic. global & $0.75 * *$ & $0.42^{* *}$ & $0.47^{* *}$ & - & & & & & \\
\hline 5. PwMS: Continue medication & $0.60 * *$ & 0.21 & $0.45 * *$ & $0.65^{* *}$ & - & & & & \\
\hline 6. PwMS: Self-rated health & $0.6 I^{* *}$ & 0.15 & $0.40 * *$ & $0.58 * *$ & $0.30 * *$ & - & & & \\
\hline 7. Physician: Response to medication & $0.50 * *$ & 0.08 & $0.26 * *$ & $0.45^{* *}$ & $0.23^{*}$ & $0.44 * *$ & - & & \\
\hline 8. Physician: Health status & $0.57^{* *}$ & 0.13 & $0.38 * *$ & $0.47 * *$ & $0.39 * *$ & $0.53^{* *}$ & $0.81 * *$ & - & \\
\hline 9. Physician: EDSS & $-0.58^{* *}$ & -0.10 & $-0.28 * *$ & $-0.51 * *$ & $-0.27^{*}$ & $-0.55 * *$ & $-0.64^{* *}$ & $-0.54^{* *}$ & - \\
\hline
\end{tabular}

Notes: *Significant at 0.05 level. **Significant at 0.01 level.

Abbreviations: Medic., medication; EDSS, Expanded Disability Status Scale. 
with global satisfaction, satisfaction with effectiveness and convenience, but not with the perception of side effects. These results are consistent with a previous study which directly assessed satisfaction and adherence and found them associated. ${ }^{15}$

This pilot study is limited by a small sample of PwMS from a single clinic. The small $\mathrm{N}$ hindered examining concordance by type of DMT, mode of administration, previous history on DMT or disease subtype. The reliance on a single clinic has circumscribed the findings and a multi-center study is a preferred course forward, eg. ${ }^{16}$ In spite of these limitations, the results point to a new direction of research: studying personalized medicine via the concordance between healthcare professionals and people with chronic diseases on treatmentrelated perceptions and decisions. The concordance may span from knowledge on medication to preferences and values placed on different outcomes. ${ }^{17}$ Discussion on these issues between the physician and PwMS could contribute to higher concordance on complex decisions and less decisional conflict, and subsequently foster higher engagement, self-management, satisfaction and medication adherence ${ }^{15}$ along with loops of feedback between the patient and the physician, augmenting a patient-physician alliance and personalizing the treatment. ${ }^{18}$

\section{Ethics}

This study was conducted in accordance with the Declaration of Helsinki.

\section{Funding}

This work used no external funding.

\section{Disclosure}

Dr. Miller has served on the scientific advisory board, and received personal compensation for consulting and/or speaking activities and/or honoraria and/or received grant support for research from: Avanir Pharmaceuticals; BayerSchering Pharma; Biogen Idec; Mapi Pharma; Medison Pharma Ltd.; Merck Serono; Novartis; Sanofi-Genzyme; and Teva Pharmaceutical Industries Ltd. The authors report no other conflicts of interest in this work.

\section{References}

1. Yeandle D, Rieckmann P, Giovannoni G, Alexandri N, Langdon D. Patient power revolution in multiple sclerosis: navigating the new frontier. Neurol Ther. 2018;7(2):179-187. doi:10.1007/s40120-018-0118-8
2. Kasper J, Köpke S, Mühlhauser I, Nübling M, Heesen C. Informed shared decision making about immunotherapy for patients with multiple sclerosis (ISDIMS): a randomized controlled trial. Eur J Neurol. 2008;15(12):1345-1352. doi:10.1111/j.1468-1331.2008.02313.x

3. Colligan E, Metzler A, Tiryaki E. Shared decision-making in multiple sclerosis. Mult Scler. 2017;23(2):185-190. doi:10.1177/ 1352458516671204

4. Poulos C, Wakeford C, Kinter E, Mange B, Schenk T. Patient and physician preferences for multiple sclerosis treatments in Germany: a discrete-choice experiment study. Mult Scler J Exp Transl Clin. 2020;6(1):1-14. doi:10.1177/2055217320910778

5. Filippi M, Bar-Or A, Piehl F, et al. Multiple sclerosis. Nat Rev Dis Prim. 2018;4(1):1-27. doi:10.1038/s41572-018-0041-4

6. Teter B, Agashivala N, Kavak K, Chouhfeh L, Hashmonay R, Weinstock-Guttman B. Characteristics influencing therapy switch behavior after suboptimal response to first-line treatment in patients with multiple sclerosis. Mult Scler J. 2014;20(7):830-836. doi: $10.1177 / 1352458513513058$

7. Koudriavtseva T, Onesti E, Pestalozza IF, Sperduti I, Jandolo B. The importance of physician-patient relationship for improvement of adherence to long-term therapy: data of survey in a cohort of multiple sclerosis patients with mild and moderate disability. Neurol Sci. 2012;33(3):575-584. doi:10.1007/s10072-011-0776-0

8. Kremenchutzky M, Walt L. Perceptions of health status in multiple sclerosis patients and their doctors. Can J Neurol Sci. 2013;40 (2):210-218. doi:10.1017/S0317167100013755

9. Rothwell PM, McDowell Z, Wong CK, Dorman PJ. Doctors and patients don't agree: cross sectional study of patients' and doctors' perceptions and assessments of disability in multiple sclerosis. $\mathrm{Br}$ Med J. 1997;314:1580-1583. doi:10.1136/bmj.314.7094.1580

10. Heesen C, Kleiter I, Nguyen F, Schaffler N, Kasper J, Kopke S. Risk perception in natalizumab-treated multiple sclerosis patients and their neurologists. Mult Scler. 2010;16(12):1507-1512. doi:10.1177/ 1352458510379819

11. Zettl UK, Bauer-Steinhusen U, Glaser T, Hechenbichler K, Hecker M. Comparative evaluation of patients' and physicians' satisfaction with interferon beta-1b therapy. BMC Neurol. 2016;16 (1):181. doi:10.1186/s12883-016-0705-1

12. Atkinson MJ, Sinha A, Hass SL, et al. Validation of a general measure of treatment satisfaction, the Treatment Satisfaction Questionnaire for Medication (TSQM), using a national panel study of chronic disease. Health Qual Life Outcomes. 2004;2(1):12. doi:10.1186/1477-7525-2-12

13. Kurtzke JF. Rating neurologic impairment in multiple sclerosis: an expanded \ndisability status scale (EDSS). Neurology. 1983;33 (11):1444-1452. doi:10.1212/WNL.33.11.1444

14. Kantor D, Bright JR, Burtchell J. Perspectives from the patient and the healthcare professional in multiple sclerosis: social media and patient education. Neurol Ther. 2018. doi:10.1007/s40120-017-0087-3

15. Glanz BI, Musallam A, Rintell DJ, Chitnis T, Weiner HL, Healy BC. Treatment satisfaction in multiple sclerosis. Int J MS Care. 2014;16 (2):68-75. doi:10.7224/1537-2073.2013-021

16. Haase R, Kullmann JS, Ziemssen T. Therapy satisfaction and adherence in patients with relapsing-remitting multiple sclerosis: the THEPA-MS survey. Ther Adv Neurol Disord. 2016;9(4):250-263. doi: $10.1177 / 1756285616634247$

17. Lancsar E, Louviere J. Conducting discrete choice experiments to inform healthcare decision making: a user's guide. Pharmacoeconomics. 2008;26(8):661-677. doi:10.2165/00019053200826080-00004

18. Lejbkowicz I, Caspi O, Miller A. Participatory medicine and patient empowerment towards personalized healthcare in multiple sclerosis. Expert Rev Neurother. 2012;12(3):343-352. doi:10.1586/ern.11.161 


\section{Publish your work in this journal}

Patient Preference and Adherence is an international, peer-reviewed, open access journal that focusing on the growing importance of patient preference and adherence throughout the therapeutic continuum. Patient satisfaction, acceptability, quality of life, compliance, persistence and their role in developing new therapeutic modalities and compounds to optimize clinical outcomes for existing disease

states are major areas of interest for the journal. This journal has been accepted for indexing on PubMed Central. The manuscript management system is completely online and includes a very quick and fair peer-review system, which is all easy to use. Visit http:// www.dovepress.com/testimonials.php to read real quotes from published authors. 